PAPER • OPEN ACCESS

\section{Design of high-quality reflectors for vertical III-V nanowire lasers on $\mathrm{Si}$}

To cite this article: Xin Zhang et al 2022 Nanotechnology 33035202

View the article online for updates and enhancements.
You may also like

- In Situ TEM Study on Au Mediated Growth
$\frac{\text { of } \mathrm{NiSi}_{2} \text { in Si Nanowire: A Vapor-Liquid- }}{\mathrm{Solid}_{2} \text { nalogy }}$
$\frac{\text { Wei Tang, S Tom Picraux, Xiaohua Liu et }}{\text { al. }}$
- $\frac{\text { (Invited) Orientation Dependent GaAs }}{\text { Nanowire Schottky Solar Cells with } 16 \%}$
$\frac{\text { Efficiency }}{\text { Ning Han and Johnny C Ho }}$
- Three-Dimensional Arrays of Lead Halide
$\frac{\text { Perovskite Nanowire Based Image }}{\text { Sensors }}$
Zhiyong Fan, Leilei Gu, Mohammadmahdi
Tavakoli et al.




\title{
Design of high-quality reflectors for vertical III-V nanowire lasers on Si
}

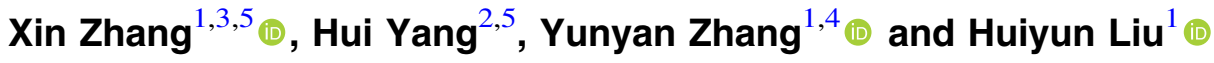 \\ ${ }^{1}$ Department of Electronic and Electrical Engineering, University College London, London WC1E 7JE, \\ United Kingdom \\ ${ }^{2}$ Department of Materials, Imperial College London, Exhibition Road, London SW7 2AZ, United \\ Kingdom \\ ${ }^{3}$ Faculty of Electrical Engineering and Computer Science, Ningbo University, Ningbo 315211, People's \\ Republic of China \\ ${ }^{4}$ Department of Physics, Universität Paderborn, Warburger Straße 100, D-33098, Paderborn, Germany \\ E-mail: yunyan.zhang@uni-paderborn.de
}

Received 14 June 2021, revised 3 September 2021

Accepted for publication 12 October 2021

Published 29 October 2021

\begin{abstract}
Nanowires (NWs) with a unique one-dimensional structure can monolithically integrate highquality III-V semiconductors onto Si platform, which is highly promising to build lasers for $\mathrm{Si}$ photonics. However, the lasing from vertically-standing NWs on silicon is much more difficult to achieve compared with NWs broken off from substrates, causing significant challenges in the integration. Here, the challenge of achieving vertically-standing NW lasers is systematically analysed with III-V materials, e.g. $\operatorname{GaAs}(\mathrm{P})$ and $\operatorname{InAs}(\mathrm{P})$. The poor optical reflectivity at the $\mathrm{NW} / \mathrm{Si}$ interface results severe optical field leakage to the substrate, and the commonly used $\mathrm{SiO}_{2}$ or $\mathrm{Si}_{2} \mathrm{~N}_{3}$ dielectric mask at the interface can only improve it to $\sim 10 \%$, which is the major obstacle for achieving low-threshold lasing. A NW super lattice distributed Bragg reflector is therefore proposed, which is able to greatly improve the reflectivity to $>97 \%$. This study provides a highly-feasible method to greatly improve the performance of vertically-standing NW lasers, which can boost the rapid development of Si photonics.
\end{abstract}

Keywords: nanowire, laser, vertically standing, optical leakage, super lattice distributed Bragg reflector

(Some figures may appear in colour only in the online journal)

\section{Introduction}

Semiconductor nanowires (NWs) lasers have a strong optical mode confinement in a very small volume, allowing lasing with diameters of a few hundred nanometers and length of a few microns [1-4]. Their small-size, low-power features endow them a wide range of novel applications [5], including Si photonics that with a big market [6]. Si photonics using

5 These authors contributed equally to this work.

Original content from this work may be used under the terms of the Creative Commons Attribution 4.0 licence. Any further distribution of this work must maintain attribution to the author(s) and the title of the work, journal citation and DOI. optical signals offers huge advantages in speed and consumption-energy saving compared with currently-used copperwire technology using electrical signals [7, 8]. However, Si has an indirect band gap and hence low light emitting efficiency. The lack of high-quality low-cost lasers as the signal sources on Si chips is the bottleneck of this technology. III-V materials have a direct-bandgap and therefore good optical properties, which are ideal for making high-efficiency lasers. However, when growing III-V thin-film components onto silicon, it is difficult to balance crystal quality, uniformity and area of selective growth, due to the large lattice, thermal properties, and atomic polarity differences [9-11]. When in the form of NWs, high-quality III-V materials can be monolithically grown on a Si platform [12-14]. This enables the fabrication of high-quality photonics light sources for low-cost, ultra-high 

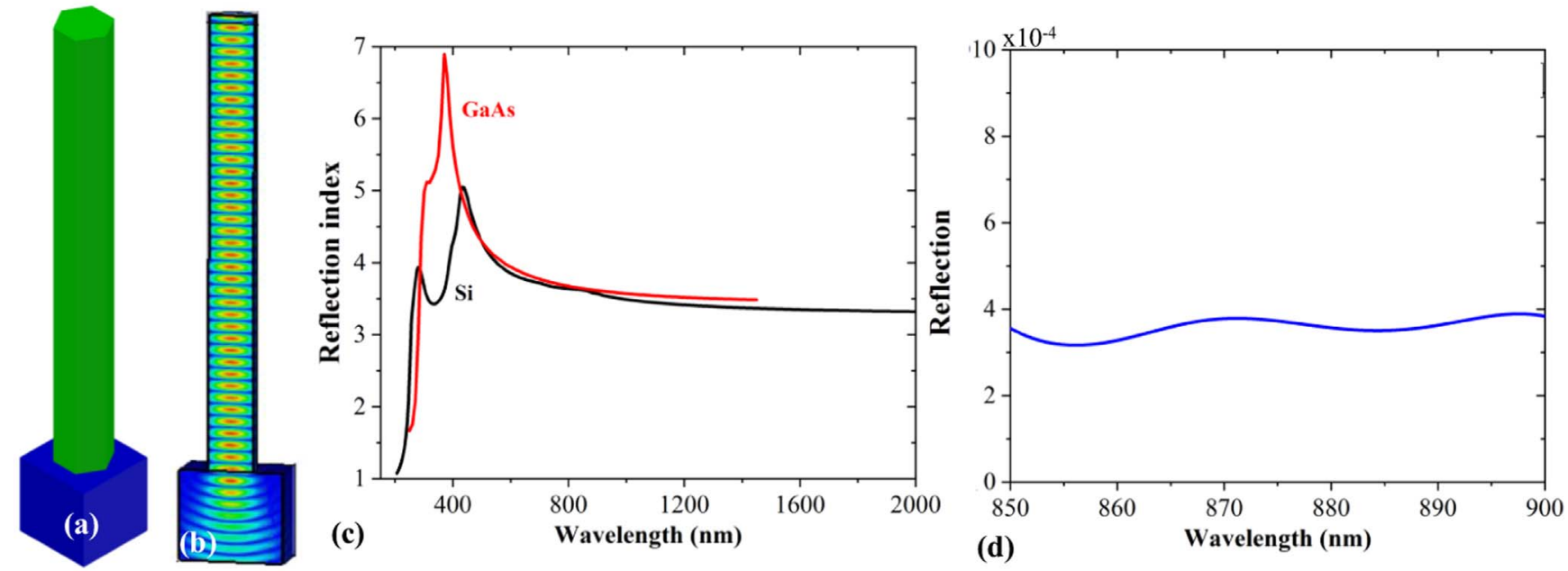

Figure 1. The light propagation and transmission behavior of a GaAs NW standing directly on Si without any special interface structure to enhance the light reflection. (a) Illustration of the structure. (b) Light-field distribution at $870 \mathrm{~nm}$ of (a). (c) Wavelength-dependent refractionindex difference between GaAs and Si. (d) Wavelength-dependent reflection at the NW/Si interface.

density integration on $\mathrm{Si}$, solving one of the major challenges that have been limiting the Si photonics [15-19].

Whilst optically-pumped NW lasers have been widely demonstrated [20-24], majority of them were broken off from their original substrate and transferred to holder substrates. In this structure, both of the NW end facets are exposed to air with a low refractive-index $(n=\sim 1)$. The large refractiveindex difference at the interface is beneficial for reflecting light back into the resonant cavity and form inverted carrier distribution. Despite the simplicity of achieving lasing with horizontal structure, NW lasers in vertically-standing structure are more desirable in most occasions due to the advantages, including massive and large-area integration, accurate allocation of NW lasers on the Si chip, simplified fabrication processes, and suitable for 3D circuit design.

However, to achieve lasing with vertically-standing structure is much more challenging, and there are only few papers on optically-pumped vertically-standing NW lasers so far [25-27]. As it has been reported, the internal quantum efficiency of the vertical NW lasers is only $16.8 \%$ [26]. This can cause enormous challenges for the fabrication of electrically-pumped lasers. One possible reason could be the similar refractive-index difference between the Si substrates and III-V materials (e.g. $n_{\mathrm{Si}} / n_{\mathrm{GaAs}}=\sim 3.63 / \sim 3.59$ at $870 \mathrm{~nm}$ ) that can cause severe optical-field leakage from the NW into the substrate and greatly degrade the quality of the optical resonant cavity. Therefore, the detailed knowledge on the causes and how to further improve performance of NW lasers are urgently needed.

In traditional thin-film devices, the super lattice (SL) distributed Bragg reflectors (DBRs) are widely used to provide strong light reflection [28, 29]. To build a high-quality DBR, material combinations with a large refractive-index difference is preferred. In the traditional thin-film growth, the strict requirement of small strain between epi-layers and substrates excludes a significant material combinations that can provide large refractive-index differences [30]. NWs with a unique one-dimensional structure and a small cross section can provide very efficient lateral and axial strain relaxation, which can offer more advantages in this structure construction and allow various material combinations that are not allowed in thin-film structures. There have been reports on defect-free heterojunctions in NWs with large lattice mismatches, e.g. InAs/InP, InAs/InSb, GaAs/GaP [31-33]. Besides, the use of III-V materials consisting two group-V elements can produce heterojunctions with atomic sharpness, which is beneficial for achieving abrupt refractive-index change $[31,34,35]$. All these advantages can be used to construct high-quality SL DBR inside NWs. Although there have been some use of SL DBR inside NWs [36-39], there is however no report is given on the topic of vertical-standing single NW lasers so far as we know.

In this work, the light propagation and transmission behavior in NWs vertically-standing on Si has been systematically studied by numerical modeling. The severe light leakage is found from $\mathrm{NWs}$ to the $\mathrm{Si}$ substrate even with the presence of $\mathrm{SiO}_{2}$ and $\mathrm{Si}_{2} \mathrm{~N}_{3}$. A SL DBRs structure is proposed, which can effectively solve this issue.

\section{Experimental section}

The software used here is Comsol Multiphysics based on finite elements method. Based on the radio frequency module integrated in this software, the models of NW lasers were built based on the observation from our experiments [40, 41], and the inner light transmission field inside NWs was simulated. This research started with $\mathrm{GaAs}(\mathrm{P}) \mathrm{NW}$ lasers, because majority of reports in literature are GaAs-based and the obtained knowledge can also be used to other material systems. The NW laser structure can be seen in figure 1(a). The GaAs NW is standing vertically on a $\mathrm{Si}$ substrate with a diameter of $\sim 500 \mathrm{~nm}$ and length of $\sim 10 \mu \mathrm{m}$, acting as both the gain material and the resonant cavity. The choose of 


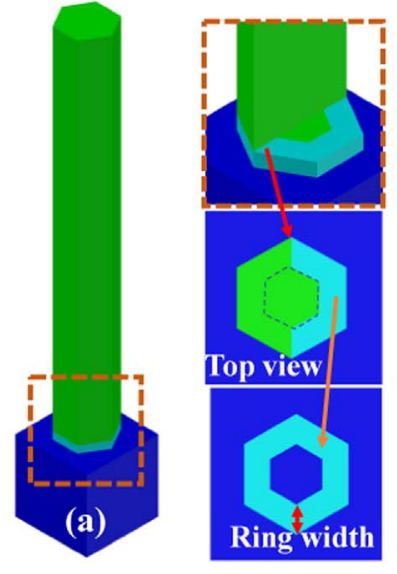

(b)
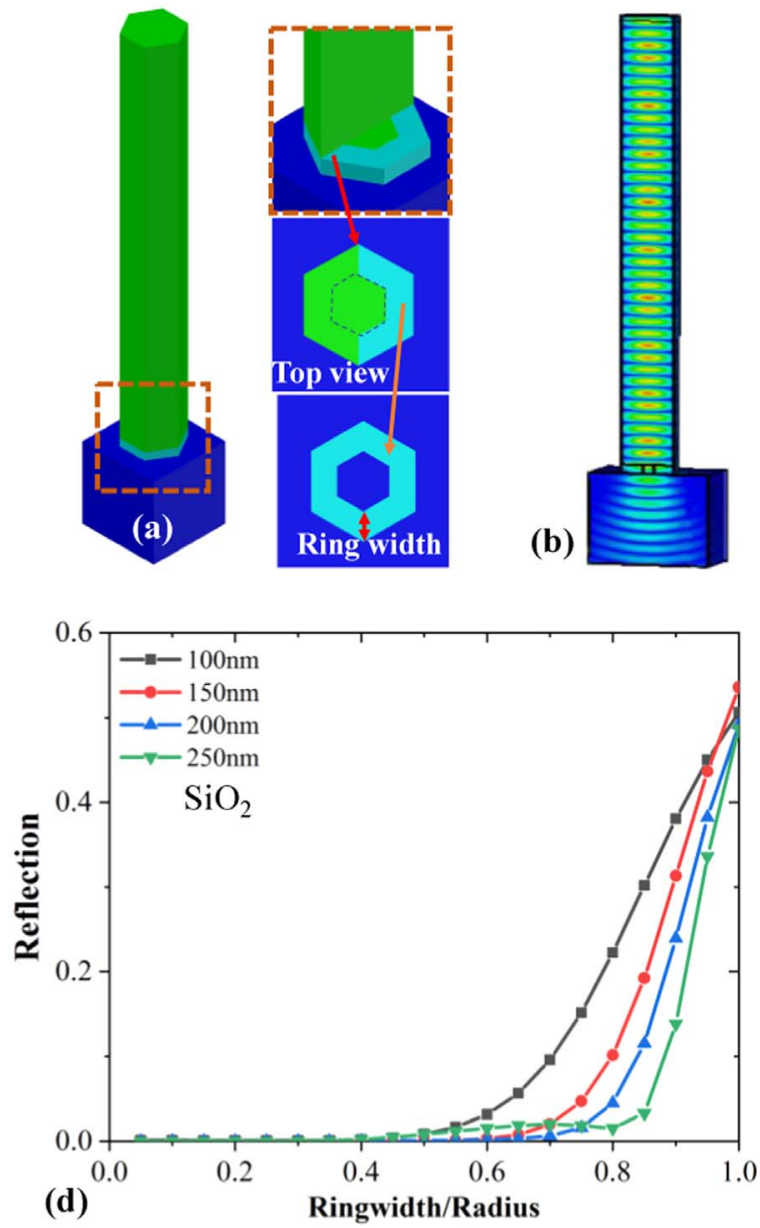
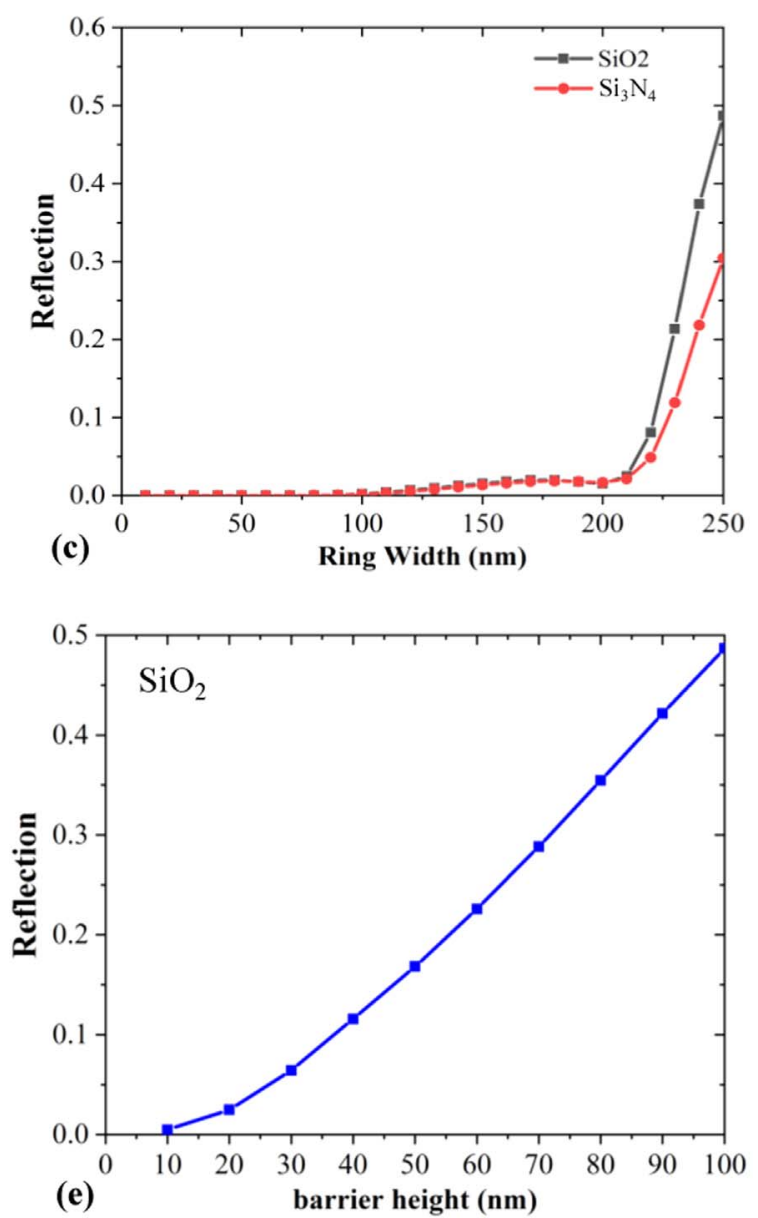

Figure 2. The light propagation and transmission behaviour of a GaAs NW (500 nm) standing directly on Si with a hexagon ring dielectric layer to enhance the light reflection. (a) Illustration of the dielectric ring reflector. (b) Light-field distribution at $870 \mathrm{~nm}$ of the GaAs NW with a $230 \mathrm{~nm}$ width $100 \mathrm{~nm}$ thick $\mathrm{SiO}_{2}$ interface layer. (c) Ring-width-dependent reflection of a GaAs NW with a $100 \mathrm{~nm}$ thick of $\mathrm{SiO}_{2}$ or $\mathrm{Si}_{3} \mathrm{~N}_{4}$ layer. (d) Influence of $\mathrm{SiO}_{2}$ ring width/radius ratio on the reflection of NW of different size. (e) $\mathrm{SiO}_{2}$ Dielectric thickness-dependent reflection of a GaAs NW with a $250 \mathrm{~nm}$ width $\mathrm{SiO}_{2}$ interface layer.

$500 \mathrm{~nm}$ diameter is due to the low loss at this size [42]. The center wavelength of laser is selected to be $870 \mathrm{~nm}$ which is the GaAs emission wavelength at the room temperature. We assume the laser beams with the same intensity propagating from the $\mathrm{NW}$ to the $\mathrm{NW} / \mathrm{Si}$ interface and integrate the reflection energy right above the interface.

\section{Results and discussion}

GaAs NWs are commonly grown directly on Si substrates without special interface structure to enhance the light reflection, which is shown in figure 1(a). As can be seen from the light-field distribution shown in figure 1(b), there is severe light energy leakage from the NW into the substrate. The refraction-index difference at the $\mathrm{NW} / \mathrm{Si}$ interface is very small for the wavelength $>500 \mathrm{~nm}$ shown in figure 1(c). As a result, the reflection at the interface is $\sim 4 \times 10^{-5}$ at the wavelength $850-890 \mathrm{~nm}$ (figure $1(\mathrm{~d})$ ). This phenomenon is highly beneficial for constructing tandem photovoltaics, such as the III-V NW/Si two junction solar cells. As demonstrated by Diedenhofen et al [43], more than $90 \%$ of the photons, with the energy lower than the InP absorption, were coupled into the underlying substrate by the InP NWs. However, it brings big challenges in the formation of proper resonant cavities for lasers with this configuration. Therefore, proper structure design is needed to reduce light leaking into the substrate.

$\mathrm{Si}_{3} \mathrm{~N}_{4}(n=\sim 2.347$ at $870 \mathrm{~nm})$ and $\mathrm{SiO}_{2}(n=\sim 1.44$ at $870 \mathrm{~nm}$ ) are widely used as the pattern mask to do the positioned NW growth on Si substrate [13], and they have a much lower refractive-index compared with III-Vs (e.g. $n_{\mathrm{GaAs}}=$ $\sim 3.5$ at $870 \mathrm{~nm}$ ). Thus, they can be potentially used to enhance the light reflection at the interface. A hexagonal ring-shaped dielectric reflector is therefore studied here (figure 2(a)). It needs to be mentioned that the inner hole is normally circular in practice due to the resolution limitation in the patterned substrate fabrication processes. The ring hole in the centre of the reflector is the opening for the NW growth with substrate lattice template. As can be seen from the lightfield distribution in the NWs in figure 2(b), the optical field leakage into the substrate reduces compared with figure 1(b) when the dielectric layer with a thickness of $100 \mathrm{~nm}$ is introduced at the interface. Both dielectric layers with a large 

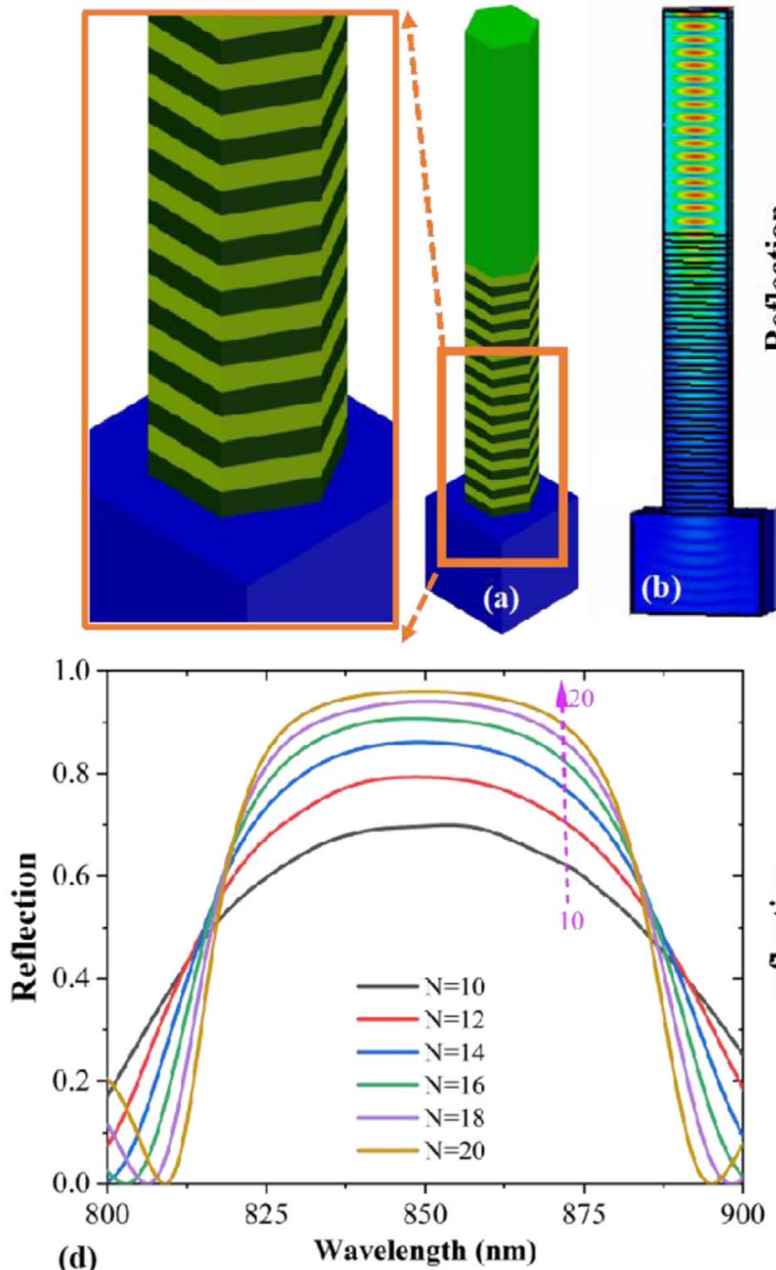

(d)
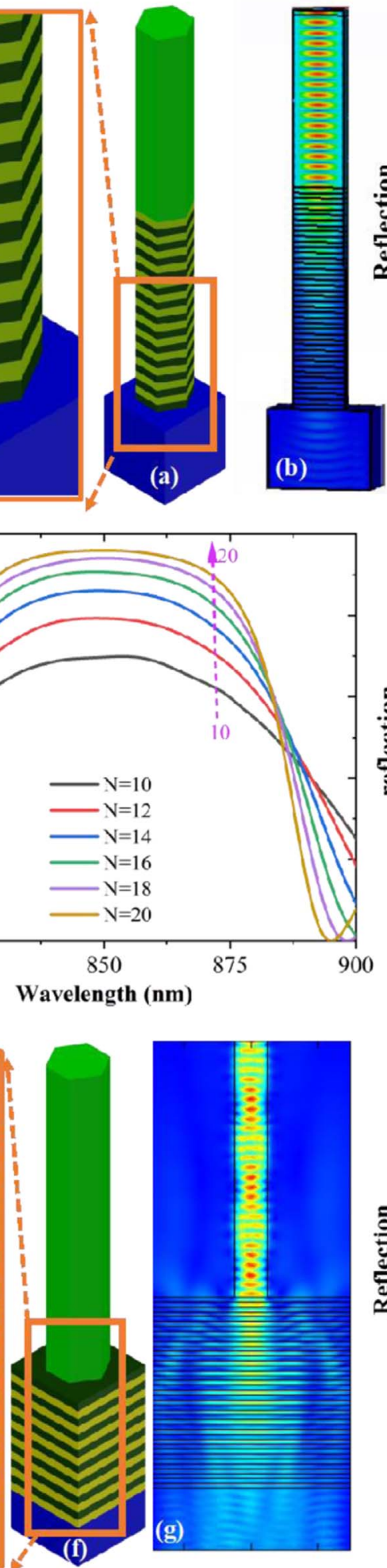
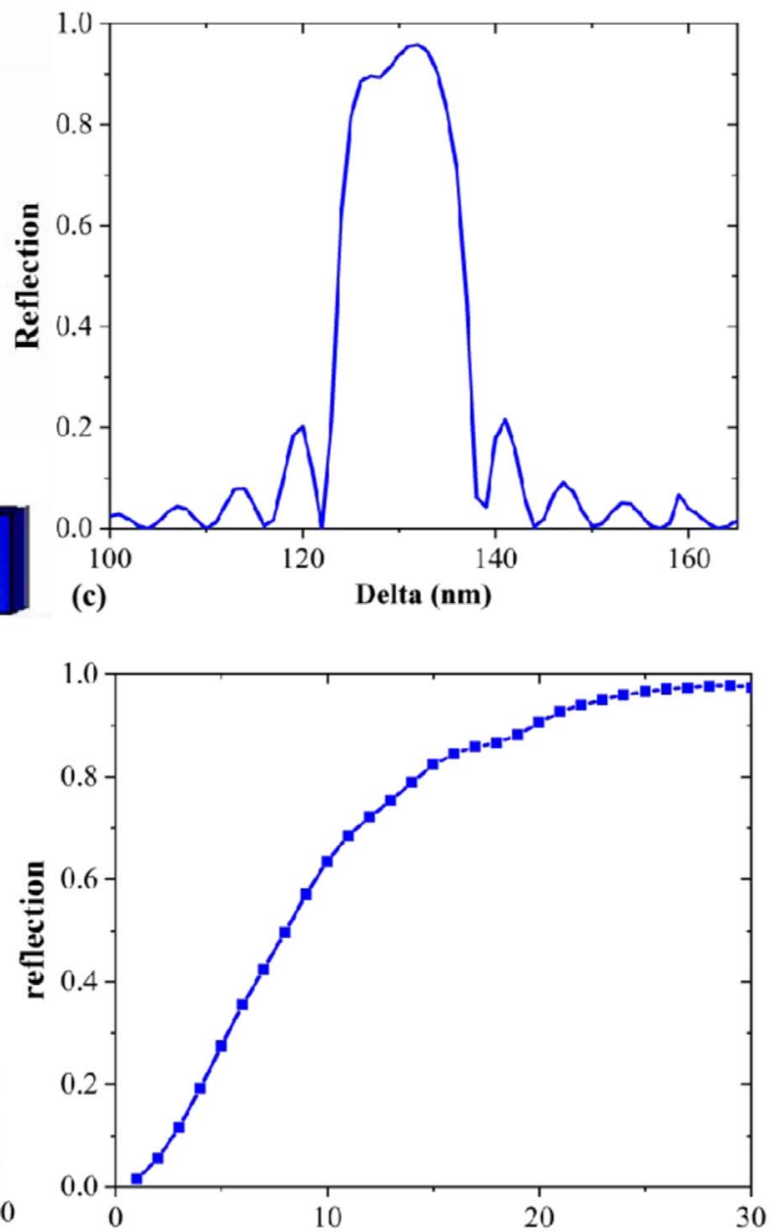

(e)

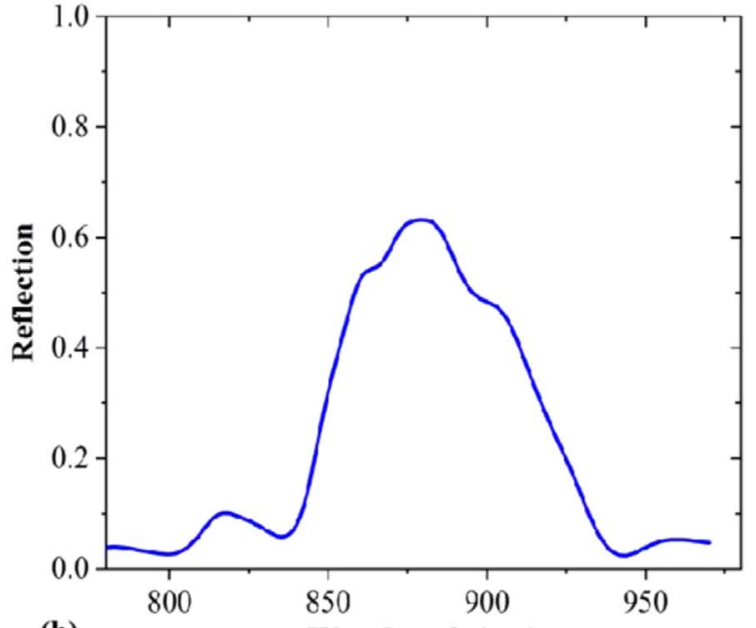

(h)

Wavelength $(\mathrm{nm})$

Figure 3. The light propagation and transmission behavior of a GaAs NW standing directly on Si with a SL DBR to enhance the light reflection. (a)-(e) are from the structure with a NW DBR reflector. (a) Illustration of the structure. (b) Light-field distribution at $870 \mathrm{~nm}$ of the NW. (c) SL-period-dependent light reflection at $870 \mathrm{~nm}$. (d) Reflection spectra of DBRs with different SL pairs. (e) SL-pairs-dependent light reflection at $870 \mathrm{~nm}$. (f)-(h) are from the structure with a substrate DBR reflector. (f) Illustration of the structure. (g) Light-field distribution at $870 \mathrm{~nm}$ of the NW. (h) Light reflection spectrum at the NW/substrate interface. 

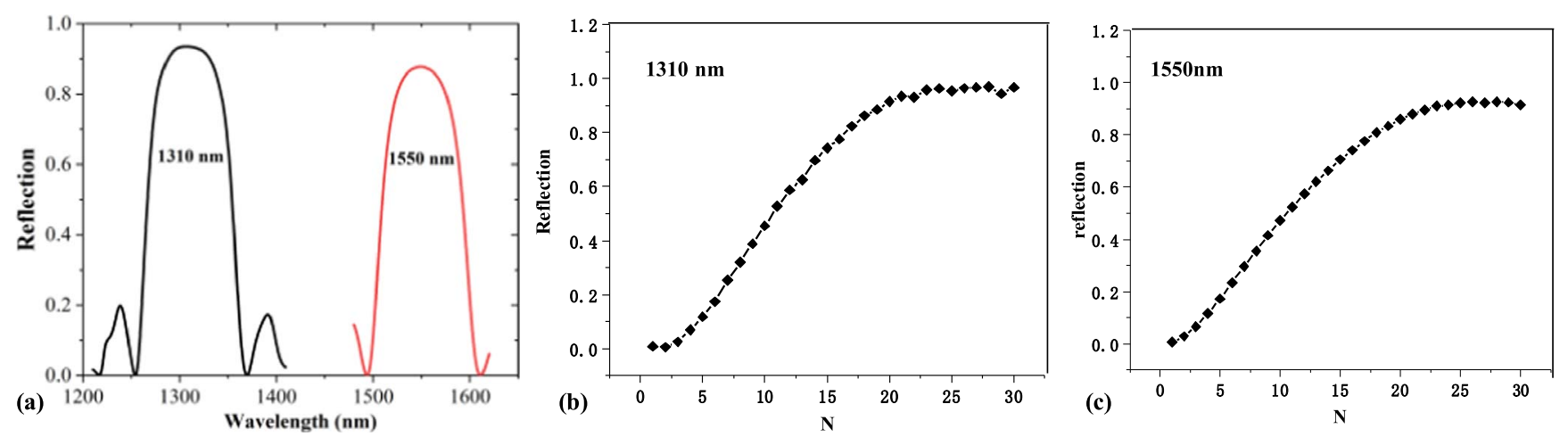

Figure 4. (a) Reflection spectra of 20-pair SL DBRs that work for 1310 and $1550 \mathrm{~nm}$ lasers. (b) and (c) Influence of SL pairs on the light reflection.

ring width, defined to be the thickness of concentric hexagon with the outer edge the same size as the NW, can increase the reflection to above $30 \%$, which is shown in figure $2(\mathrm{c})$. However, the interface reflectance is small $(<3 \%)$ for small ring widths and rises slowly with the increase of the width; only when the width is approaching the NW diameter, the reflectance rises rapidly. This phenomenon is the same for NWs of different diameters we study (figure 2(d)), which suggest that the width of dielectric needs to be at least over half the size of the NW to get more apparent reflection. It needs to be noted that the interface reflection of NWs with a $\mathrm{SiO}_{2}$ layer can be up to $67 \%$ higher than that of $\mathrm{Si}_{3} \mathrm{~N}_{4}$ layer at a large ring width, which is due to the larger index difference with the NW. Consequently, it would be better to choose $\mathrm{SiO}_{2}$ as the reflector material when building lasers. Moreover, the thickness of the dielectric layer can greatly influence the reflectance. As is shown in figure 2(e), the reflection of the NWs with a $\mathrm{SiO}_{2}$ dielectric layer grows almost linear to $\sim 50 \%$ at $100 \mathrm{~nm}$. At present, the thickness of dielectric pattern is normally between 20 and $30 \mathrm{~nm}$, which can only provide a low reflectivity of $\sim 10 \%$. We have not notice the reports using thicker patterns. More study is needed about growing on super-thick patterns, such as 100 or $200 \mathrm{~nm}$, which can however bring high challenges on both the pattern making and NW growth.

With the assumption that a $100 \mathrm{~nm}$ thick dielectric reflector between the $\mathrm{NW} / \mathrm{Si}$ interface can be easily used, the reflection can only be improved to $\sim 50 \%$ and a high percentage of the light is still lost through the substrate, causing high threshold of electrically-pumped lasers. Considering the advantages of NWs in the construction of heterojunctions by switching group-V elements as mentioned above, a $\mathrm{GaAs}_{0.1} \mathrm{P}_{0.9} / \mathrm{GaAs}\left(n_{\mathrm{GaAsP}} / n_{\mathrm{GaAs}}=3.2 / 3.59\right) \mathrm{SL}$ DBR can be introduced between NW and $\mathrm{Si}$ substrate (figure 3(a)). As can be seen from the light-field distribution in figure 3(b), most of light-field is resonating inside the NW with only little light energy leaks into the substrate. The SL period is one of the key parameters that decide the peak reflection wavelength. For a lasing wavelength of $870 \mathrm{~nm}$, a high reflectance of over $80 \%$ can be achieved with a period between 124 and $142 \mathrm{~nm}$, and the reflection reaches the maximum can be $96 \%$ at $136 \mathrm{~nm}$ (figure 3(c)). The pair of SL can also significantly influence the light reflection. As can be seem in figure $3(\mathrm{~d})$, the reflection increases rapidly with the increase of the SL pairs to 15 and gradually saturates after 20 pairs. The reflection at $870 \mathrm{~nm}$ can reach 0.96 with a 20-pair SL and 0.98 with a 30-pair SL. It needs to be mentioned that the SL DBR can have a large reflection window. For example, its reflectance can maintain above $90 \%$ between 820 and $880 \mathrm{~nm}$. Thus, the SL DBR has significant advantages over the dielectric reflectors.

The technique is well developed for growing the SL DBR on substrate surface in the form of thin-film structure. Integrating NWs directly onto the substrates with DBRs (figure 3(f)) can provide a possibility to avoid the complexity of growing DBR into NWs, which has been widely used experimentally [44-46]. Thus, the influence of the location on the reflection effect of SL DBR is also studied with the optimized DBR parameters used in figure 3(c). It needs to be mentioned that we only intend to make a direct comparison of the two structures, and assume that the lattice mis-match in the substrate DBR growth is not an issue. As can be seen from figure $3(\mathrm{~g})$, the DBR can effectively avoid the leakage of the optical field into the substrate. However, the field disperses after coming out of the NW bottom into the substrates, and a significant portion of the energy can no longer be reflected back to the NWs with a small cross section. As supported by the reflection spectrum in figure $3(\mathrm{~h})$, the energy in the NW at the peak wavelength can only maintain at $\sim 60 \%$, which is greatly reduced compared with that of the NW DBR.

The studied method is also extended to telecom communication wavelength. The NWs used here are made of InAsP. The InAs/InP lattice periods are $0.236 \mu \mathrm{m}$ for $1310 \mathrm{~nm}$ light and $0.28 \mu \mathrm{m}$ for $1550 \mathrm{~nm}$ light respectively. As can be seen in figure 4 , the SL DBR can also work well with these lasers and both of them can improve the light reflection to above $90 \%$ when the SL pairs are over 20 .

So far as we know, there is still lack of experiment report on the vertically-standing single nanowire lasers with this design. More experiment effort is needed. Besides, Heo et al [38] reported the building of quasi-film DBR reflector in the high density nanowire arrays, and increased the light reflection to above $70 \%$. This can partially support the feasibility of using our design in the vertically-standing single NW lasers. 


\section{Conclusions}

In summary, the challenge of achieving vertically-standing NW lasers on $\mathrm{Si}$ is systematically analyzed. The optical reflectivity at the GaAs NW/Si interface is $\sim 4 \times 10^{-5}$ at the wavelength $850-890 \mathrm{~nm}$, which is due to the small refraction-index difference between $\mathrm{GaAs}$ and $\mathrm{Si}$. This small reflectivity prevents the formation of acceptable optical resonant cavity for the realization of lasing. $\mathrm{SiO}_{2}$ and $\mathrm{Si}_{2} \mathrm{~N}_{3}$, with larger refraction-index differences with $\mathrm{Si}$, are widely used in the NW growth, which is however can only improve it to $\sim 10 \%$ and majority of the energy is still leaking into the substrates. To further improve the reflectivity at $\mathrm{NW} /$ substrate interfaces, the NW SL DBR is proposed, which can greatly improve the reflectance to $97 \%$, allowing the formation of high-quality optical resonant cavity. Besides, these reflectors can also be used for telecom communication wavelength $(1310 \mathrm{~nm}$ and $1550 \mathrm{~nm})$ and a high reflectance of $\sim 90 \%$ can be achieved. It needs to be mentioned that the SL growth in NWs will need a large change in growth fluxes and hence the growth environment, which can lead to the generation of stacking faults. A carefully balanced growth environment will be needed in the DBR growth [47]. This research provides useful information for the design of highquality vertically-standing NW lasers onto Si platform, which can be widely used in the integrated circuits.

\section{Acknowledgments}

The authors acknowledge the support of Leverhulme Trust, EPSRC (grant nos. EP/P000916/1, EP/P000886/1, EP/ P006973/1), and EPSRC National Epitaxy Facility.

\section{Data availability statement}

All data that support the findings of this study are included within the article (and any supplementary files).

\section{Notes}

The authors declare no competing financial interest.

\section{ORCID iDs}

Xin Zhang (i) https://orcid.org/0000-0002-6669-7037

Yunyan Zhang (1) https://orcid.org/0000-0002-2196-7291

Huiyun Liu (1) https://orcid.org/0000-0002-7654-8553

\section{References}

[1] Cui Y, Wei Q Q, Park H K and Lieber C M 2001 Nanowire nanosensors for highly sensitive and selective detection of biological and chemical species Science 293 1289-92
[2] Horth A and Quitoriano N J 2012 Novel, low-index waveguide as laser external cavity Opt. Express 20 11137-42

[3] Wang J, Gudiksen M S, Duan X, Cui Y and Lieber C M 2001 Highly polarized photoluminescence and photodetection from single indium phosphide nanowires Science 293 $1455-7$

[4] Yan R, Gargas D and Yang P 2009 Nanowire photonics Nat. Photon. 3 569-76

[5] Zhang Y, Wu J, Aagesen M and Liu H 2015 III-V nanowires and nanowire optoelectronic devices J. Phys. D: Appl. Phys. 48463001

[6] Paul D J 2009 Silicon photonics: a bright future? Electron. Lett. 45 582-4

[7] Liu M, Zhang M, Zhao W, Song C, Wang D, Li Q and Wang Z 2017 Prediction of congestion degree for optical networks based on bp artificial neural network 2017 16th Int. Conf. on Optical Communications and Networks (ICOCN) (Piscataway, NJ: IEEE) pp 1-3

[8] J Ni J and Bai X 2017 A review of air conditioning energy performance in data centers Renew. Sustain. Energy Rev. 67 625-40

[9] Fitzgerald E A and Chand N 1991 Epitaxial necking in GaAs grown on pre-pattemed Si substrates J. Electron. Mater. 20 $839-53$

[10] Chang J S, Carey K W, Turner J E and Hodge L A 1990 Compositional non-uniformities in selective area growth of GaInAs on InP grown by OMVPE J. Electron. Mater. 19 $345-8$

[11] Wang Z, Abbasi A, Dave U, De Groote A, Kumari S, Kunert B and Roelkens G 2017 Novel light source integration approaches for silicon photonics Laser Photon. Rev. 111700063

[12] Li K H, Liu X, Wang Q, Zhao S and Mi Z 2015 Ultralowthreshold electrically injected AlGaN nanowire ultraviolet lasers on Si operating at low temperature Nat. Nanotechnol. 10 140-4

[13] Zhang Y, Wu J, Aagesen M, Holm J, Hatch S, Tang M, Huo S and Liu H 2014 Self-catalyzed ternary core-shell GaAsP nanowire arrays grown on patterned Si substrates by molecular beam epitaxy Nano Lett. 14 4542-7

[14] Frost T, Jahangir S, Stark E, Deshpande S, Hazari A, Zhao C, Ooi B S and Bhattacharya P 2014 Monolithic electrically injected nanowire array edge-emitting laser on (001) silicon Nano Lett. 14 4535-41

[15] Saxena D, Mokkapati S, Parkinson P, Jiang N, Gao Q, Tan H H and Jagadish C 2013 Optically pumped roomtemperature GaAs nanowire lasers Nat. Photon. 7 963-88

[16] Fischer R, Masselink W T, Klem J, Henderson T, McGlinn T C, Klein M V, Morkoç H, Mazur J H and Washburn J 1985 Growth and properties of GaAs/AlGaAs on nonpolar substrates using molecular beam epitaxy J. Appl. Phys. 58 374-81

[17] Kim H, Lee W J, Farrell A C, Morales J S, Senanayake P, Prikhodko S V, Ochalski T J and Huffaker D L 2017 Monolithic InGaAs nanowire array lasers on silicon-oninsulator operating at room temperature Nano Lett. 17 3465-70

[18] Liang D and Bowers J E 2010 Recent progress in lasers on silicon Nat. Photon. 4 511-7

[19] Won R and Mario P 2010 Integrating silicon photonics Nat. Photon. 4 498-9

[20] Guilhabert B, Hurtado A, Jevtics D, Gao Q, Tan H H, Jagadish C and Dawson M D 2016 Transfer printing of semiconductor nanowires with lasing emission for controllable nanophotonic device fabrication ACS Nano 10 3951-8

[21] Zhang Y, Saxena D, Aagesen M and Liu H 2019 Toward electrically driven semiconductor nanowire lasers Nanotechnology 30192002 
[22] Xu W Z et al 2018 Vertically emitting indium phosphide nanowire lasers Nano Lett. 18 3414-20

[23] Stettner T et al 2016 Coaxial GaAs-AlGaAs core-multishell nanowire lasers with epitaxial gain control Appl. Phys. Lett. 108011108

[24] Tatebayashi J, Kako S, Ho J, Ota Y, Iwamoto S and Arakawa Y 2015 Room-temperature lasing in a single nanowire with quantum dots Nat. Photon. 9 501-5

[25] Mayer B, Janker L, Loitsch B, Treu J, Kostenbader T, Lichtmannecker S and Gies C 2015 Monolithically integrated high- $\beta$ nanowire lasers on silicon Nano Lett. 16 152-6

[26] Sun H, Ren F, Ng K W, Tran T T D, Li K and Chang-Hasnain C J 2014 Nanopillar lasers directly grown on silicon with heterostructure surface passivation Acs Nano 8 6833-9

[27] Chen R, Tran T T D, Ng K W, Ko W S, Chuang L C, Sedgwick F G and Chang-Hasnain C 2011 Nanolasers grown on silicon Nat. Photon. 5 170-5

[28] Mastro M A, Holm R T, Bassim N D, Eddy C R Jr, Gaskill D K, Henry R L and Twigg M E 2005 Highreflectance III-nitride distributed Bragg reflectors grown on Si substrates Appl. Phys. Lett. 87241103

[29] Huang G S, Lu T C, Yao H H, Kuo H C, Wang S C, Lin C W and Chang L 2006 Crack-free distributed Bragg reflectors incorporated with superlattices grown by metalorganic chemical vapor deposition Appl. Phys. Lett. 88061904

[30] Shi J Y, Yu L P, Wang Y Z, Zhang G Y and Zhang H 2002 Influence of different types of threading dislocations on the carrier mobility and photoluminescence in epitaxial GaN Appl. Phys. Lett. 80 2293-5

[31] Larsson M W, Wagner J B, Wallin M, Håkansson P, Fröberg L E, Samuelson L and Wallenberg L R 2006 Strain mapping in free-standing heterostructured wurtzite InAs/InP nanowires Nanotechnology 18015504

[32] Ercolani D, Rossi F, Li A, Roddaro S, Grillo V, Salviati G, Beltram F and Sorba L 2009 InAs/InSb nanowire heterostructures grown by chemical beam epitaxy Nanotechnology 20505605

[33] Zhang G, Tateno K, Gotoh H, Sogawa T and Nakano H 2010 Structural compositional and optical characterizations of vertically aligned $\mathrm{AlAs} / \mathrm{GaAs} / \mathrm{GaP}$ heterostructure nanowires epitaxially grown on Si substrate Jpn. J. Appl. Phys. 49015001

[34] Priante G, Patriarche G, Oehler F, Glas F and Harmand J C 2015 Abrupt $\mathrm{GaP} / \mathrm{GaAs}$ interfaces in self-catalyzed nanowires Nano Lett. 15 6036-41
[35] Zhang Y et al 2020 Defect-free axial GaAs/GaAsP nanowire quantum dots with strong carrier confinement Nano Lett. 215722

[36] Fu A, Gao H, Petrov P and Yang P 2015 Widely tunable distributed Bragg reflectors integrated into nanowire waveguides Nano Lett. 15 6909-13

[37] Aghaeipour M and Pettersson H 2018 Enhanced broadband absorption in nanowire arrays with integrated Bragg reflectors Nanophotonics 7 819-25

[38] Heo J, Zhou Z, Guo W, Ooi B S and Bhattacharya P 2013 Characteristics of AlN/GaN nanowire Bragg mirror grown on (001) silicon by molecular beam epitaxy Appl. Phys. Lett. 103181102

[39] Svendsen G K, Weman H and Skaar J 2012 Investigations of Bragg reflectors in nanowire lasers J. Appl. Phys. 111 123102

[40] Wu J et al 2016 Defect-free self-catalyzed GaAs/GaAsP nanowire quantum dots grown on silicon substrate Nano Lett. 16 504-11

[41] Zhang Y and Liu H 2020 Self-catalyzed GaAs (P) nanowires and their application for solar cells J. Phys. D: Appl. Phys. 53233001

[42] Saxena D, Jiang N, Yuan X, Mokkapati S, Guo Y, Tan H H and Jagadish C 2016 Design and room-temperature operation of GaAs/AlGaAs multiple quantum well nanowire lasers Nano Lett. 16 5080-6

[43] Diedenhofen S L, Janssen O T, Grzela G, Bakkers E P and Gómez Rivas J 2011 Strong geometrical dependence of the absorption of light in arrays of semiconductor nanowires ACS Nano 5 2316-23

[44] Tatebayashi J, Kako S, Ho J, Ota Y, Iwamoto S and Arakawa Y 2017 Growth of InGaAs/GaAs nanowirequantum dots on $\mathrm{AlGaAs} / \mathrm{GaAs}$ distributed Bragg reflectors for laser applications J. Cryst. Growth 468 144-8

[45] Bashar S B, Suja M, Shi W and Liu J 2016 Enhanced random lasing from distributed Bragg reflector assisted $\mathrm{Au}-\mathrm{ZnO}$ nanowire schottky diode Appl. Phys. Lett. 109192101

[46] Huang J, Monzur Morshed M, Zuo Z and Liu J 2014 Distributed Bragg reflector assisted low-threshold $\mathrm{ZnO}$ nanowire random laser diode Appl. Phys. Lett. 104131107

[47] Zhang Y, Velichko A V, Fonseka H A, Parkinson P, Davis G, Gott J A, Aagesen M, Sanchez A M, Mowbray D and Liu H 2021 Defect-free axially-stacked GaAs/GaAsP Nanowire quantum dots with strong carrier confinement Nano Lett. 21 5722-9 